\title{
Introduction to the WIAMan Biomechanics Program
}

\author{
(Published online 1 April 2021)
}

The Warrior Injury Assessment Manikin (WIAMan) program is an ongoing effort, led by the US Army Combat Capabilities Development Command Data \& Analysis Center (DEVCOM DAC), to develop the world's first test manikin with a validated injury assessment capability for underbody blast (UBB) testing of ground vehicles in Live-fire Test and Evaluation (LFT\&E).

The use of improvised explosive devices (IEDs) has been a common tactic employed by opposing forces in U.S. military conflicts over the past two decades. When activated under a vehicle, IEDs impart high-rate vertical loads to the occupants of the vehicle that can cause severe injury and death. In developing countermeasures, including improved energy attenuating mechanisms and vehicle designs, the military has previously relied on automotive crash surrogates. In particular, they have used anthropomorphic test devices (ATD) originally developed for civilian traffic safety to evaluate vehicles against UBB loading. Although the specialized ATDs utilized by the automotive industry have been proven for a variety of scenarios, they were not designed for measuring the high-rate vertical force created by UBBs or for predicting the resultant injuries to vehicle occupants.

To address this gap, in 2010 the US Army initiated the WIAMan program to design, develop, and validate a specialized ATD to measure and evaluate the risk of skeletal injuries during high-rate vertical loading to seated ground vehicle occupants. The WIAMan biomechanics program includes nationally recognized biomechanics experts from leading research institutions to support the design of the WIAMan ATD and to develop the associated WIAMan ATD injury assessment capability. The first Generation of the WIAMan (WIAMan Gen 1) ATD was manufactured by Diversified Technical Systems in 2018 (Fig. 1). Future Warfighter protection systems and vehicles will be better informed as a result of the WIAMan program.

The WIAMan biomechanics program has primarily focused on the following anatomic regions: head-neck, lumbar spine, pelvis, and lower extremities (Fig. 2). These components have the highest respective preva- lence and/or severity of injury in UBB events in theater, as loads travel upward through the floor and seat and into the body. Biomechanical testing was performed in controlled environments using test rigs designed to emulate impact similar to a UBB event, for impacts to both the whole body and isolated component body regions. Component level tests remove compounding effects from adjacent anatomical regions and allow a focused investigation of anatomical regions of interest. Whole body tests are conducted to understand the relationship between each of the components of interest, and to guide subsystem and component testing. The majority of test data has centered around "90-90-90" hip-knee-ankle posture angles. This nominal posture is representative of the average posture of a midsize male Soldier, seated with a vertical back and a horizontal seat pan. The program is now beginning to explore the impact of posture change on injury mechanism, probability, and severity.

There have been three phases to the biomechanics effort:

Biofidelity response corridors (BRCs): The first phase of the biomechanics efforts focused on developing BRCs to support the design of the WIAMan Gen 1 ATD. These tests were low-impact, non-injurious tests conducted to provide insight into the biomechanical response of the anatomical components and the biofidelity of the WIAMan ATD.

Human Injury Probability Curves (HIPCs): The second phase of the biomechanics effort was the development of HIPCs. For WIAMan, HIPCs allow researchers to predict the probability and severity of injury that will result from the impact of an accelerative vertical loading event to the human body. For HIPC generation, non-injurious and injurious tests were conducted on isolated anatomical components. The corresponding biomechanical test data was processed, and utilizing survival analysis techniques, the statistical relationship between the probability of an injury and a single biometric or combination of biometrics measured was then calculated.

Injury Assessment Reference Curves (IARCs): The third phase of the biomechanics effort was to relate human injury risk as characterized in the HIPCs to the 


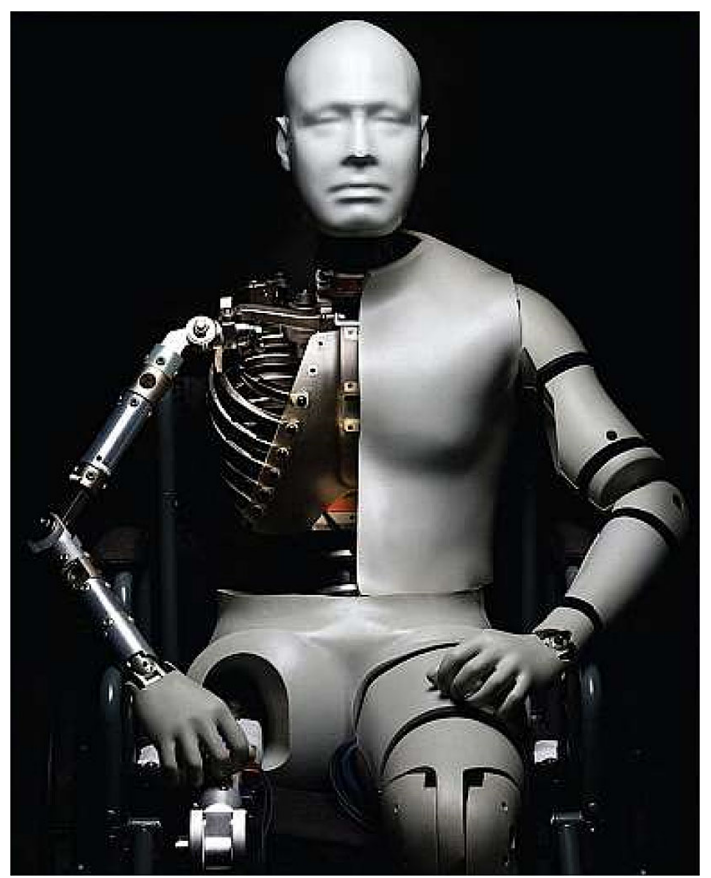

FIGURE 1. Generation 1 WIAMan.

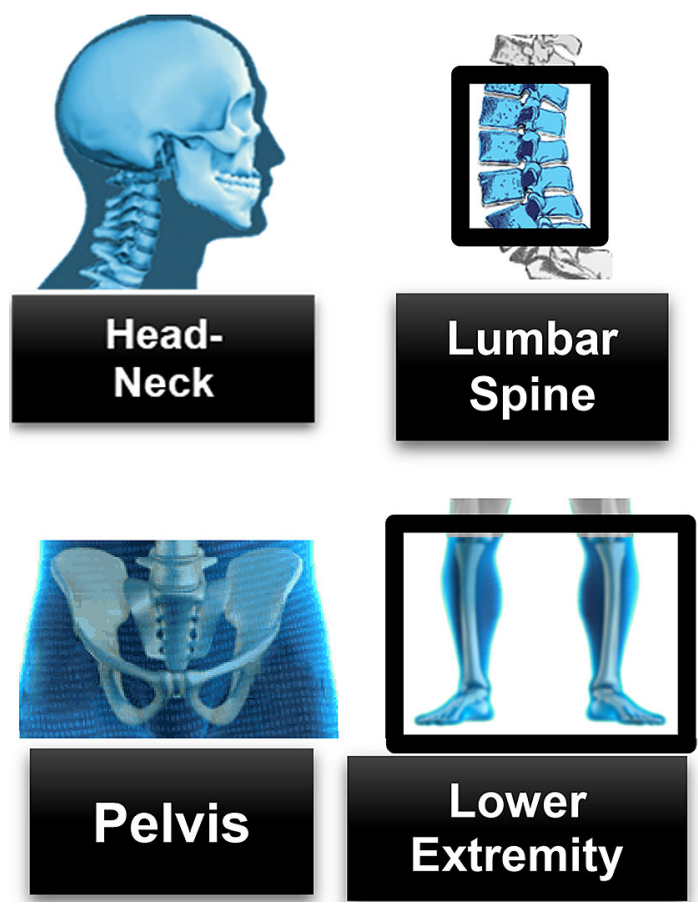

FIGURE 2. Anatomical regions of interest.

measured sensor response in the WIAMan ATD, developing IARCs for each injury region. This was achieved through match-paired testing and specialized data analysis efforts for the WIAMan Gen 1 ATD, conducted at both component and full-body levels. The IARCs will unfortunately not be featured in this journal special addition as they are still under review by the US Army.

A finite element model (FEM) of the WIAMan ATD has also been used to supplement the biomechanics effort. The computational model was initially developed to support the evaluation of material and design considerations for strength and biofidelity of the ATD at the program's onset. Since then, it has been matured and validated against the aforementioned experimental data to support biomechanics test planning, and to potentially serve as an injury assessment tool.

As a whole, the biomechanical achievements in 10 years of the WIAMan biomechanics program has been staggering. More than 500 component tests and over 70 whole body tests have been completed in the development of 800 BRCs, and created more than 15 HIPCs and IARCs, to accompany the WIAMan ATD for use in LFT\&E assessments. In comparison, similar efforts in the automotive industry required more than 20 years to obtain comparable results. This research has involved the collaboration of injury biomechanics experts across the United States, including contributions from the Johns Hopkins University Applied Physics Laboratory, the Medical College of Wisconsin, Duke University, the University of Virginia, Wayne State University, the University of Michigan Transportation Research Institute, Wake Forest University, Virginia Tech, and Ohio State University. We are pleased to be able to share this work with the biomechanics community through this Special Edition journal, focusing on the biofidelity analysis and HIPC testing and creation which can be presented in a public forum.

\section{Kathryn Loftis and Kate Sandora \\ Combat Capabilities Development \\ Command Data and Analysis Center}

DAVID DREWRY III (iD

Johns Hopkins University Applied Physics

Laboratory

Electronic mail: David.g.drewry@jhuapl.edu

Publisher's Note Springer Nature remains neutral with regard to jurisdictional claims in published maps and institutional affiliations. 